\title{
Análise de fatores e regressão bissegmentada no estudo da adaptabilidade e estabilidade de cenoura
}

\author{
Agnaldo Donizete Ferreira de Carvalho ${ }^{1}$, Giovani Olegario da Silva ${ }^{1}$, \\ Ricardo Borges Pereira ${ }^{1}$, Jadir Borges Pinheiro ${ }^{1}$ \\ http://dx.doi.org/10.1590/0034-737X201461060007
}

\begin{abstract}
RESUMO
O objetivo deste trabalho foi avaliar a estabilidade e a adaptabilidade de oito genótipos de cenoura (três cultivares e cinco populações), em 17 ambientes, nas regiões nordeste, centro-oeste, sudeste e sul do Brasil, nos anos agrícolas 2009/10, 2010/11 e 2011/12. Os experimentos foram conduzidos entre novembro e janeiro de cada ano, conforme o calendário agrícola dos produtores. A parcela experimental foi composta de área igual a $1,0 \mathrm{~m}^{2}, \mathrm{em}$ delineamento de blocos ao acaso, com três repetições. Os tratos culturais foram similares aos usados pelos produtores. Na colheita, as raízes comercializáveis foram quantificadas e os valores transformados para t ha ${ }^{-1}$. Realizouse a análise de variância e, posteriormente, a análise de fatores e a análise de regressão bissegmentada, visando ao estudo de estabilidade e adaptabilidade. Nenhum genótipo apresentou adaptabilidade ampla. A análise de fatores conseguiu agrupar menos de 50\% dos ambientes avaliados. O genótipo ideal não foi encontrado pela análise de regressão. O cultivar 'BRS Planalto' foi o mais adaptado, pelo método da regressão bissegmentada, enquanto, pela análise de fatores, 'Brasília', CNPH-600 e CNPH-589 mostraram-se mais adaptados. Os resultados obtidos por essas metodologias demonstram a dificuldade de recomendação de um cultivar para todas as regiões produtoras e que programas regionalizados devem ser iniciados. A utilização dos dois métodos associados permite a tomada de decisões mais segura acerca do comportamento dos genótipos de cenoura.
\end{abstract}

Palavras-chave: Daucus carota L., cultivares, programa de melhoramento, metodologia, interação genótipo x ambiente.

\section{ABSTRACT}

\section{Analysis of factors and bissegmented regression in the study of adaptability and stability in carrots}

The objective of this study was to evaluate the stability and adaptability of eight genotypes of carrots (three cultivars and five populations) in 17 environments in the Northeast, Midwest, Southeast and Southof Brazil in the crop years 2009/10, 2010/11 and 2011/12. The experiments were grown from November to January of each year according to the farmers' crop calendar. The plot was composed of an area with $1.0 \mathrm{~m}^{2}$ in a randomized block design with three replications. The cultural practices were the same used by the farmers. At harvest, the marketable roots were quantified and the values converted to tha ${ }^{-1}$. Analysis of variance was performed and after that, the factor analysis and bissegmented regression analysis to study the stability and adaptability. None of the genotypes showed wide adaptability. Factor analysis was able to cluster less than $50 \%$ of the evaluated environments. The ideal genotype was not found by regression analysis. The 'BRS Planalto' cultivar was the most adaptedby the bissegmented regression method while 'Brasília' CNPH-589 and CNPH-600 were the most adapted by factor analysis. The results obtained by these methods demonstrate the difficulty of recommending a cultivar for all producing regions and regionalized programs should be initiated. The use of the two associated methods allows safer decisions concerning the behavior of genotypes of carrot.

Key words: breeding, cultivars, Daucus carota L., genotype x environment interaction, methodology.

Recebido para publicação em 18/06/2013 e aprovado em 01/11/2013.

'Engenheiros-Agrônomos, Doutores. Embrapa Hortaliças, Rodovia BR 060, Quilômetro 09, 70359-970, Caixa Postal 218, Brasília, Distrito Federal, Brasil. agnaldo.carvalho@embrapa.br (autor correspondente); giovani.olegario@embrapa.br; ricardo-bordes.pereira@embrapa.br; jadir.pinheiro@embrapa.br 


\section{INTRODUÇÃO}

A cenoura ocupa lugar de destaque entre as hortaliças cultivadas no Brasil. Segundo o Anuário Brasileiro de Hortaliças (2012), a área plantada com essa raiz tuberosa foi superior a $25 \mathrm{mil}$ ha, na safra de 2010, o que gerou uma produção de mais de 760 mil toneladas de raízes comercializadas.

No Brasil, a cenoura é cultivada nas regiões sul, sudeste, centro-oeste e nordeste. Essa grande extensão territorial, com condições edafoclimáticas distintas, os diferentes níveis de investimento e formas de cultivo fazem com que o rendimento da cenoura seja variável. Essa falta de coincidência da expressão fenotípica nos diferentes ambientes, em que a cenoura é avaliada, é denominada de interação genótipos $\mathrm{x}$ ambientes (Oliveira et al., 2005).

As respostas não coincidentes dos genótipos, nas diferentes regiões, tornam difícil a recomendação de um único genótipo para a vasta extensão territorial de cultivo. Por essa razão, o melhorista precisa utilizar ferramentas que auxiliem, tanto na fase de seleção como na de recomendação dos novos cultivares (Cargnin et al., 2006).

Para a cultura da cenoura, são poucos os trabalhos visando ao estudo da interação genótipo $\mathrm{x}$ ambiente ( $\mathrm{G} x$ E). Oliveira et al. (2005) avaliaram a adaptabilidade e estabilidade de cultivares de cenoura pelo método de Eberhart \& Russel (1966), em São José do Rio PardoSP, Ponta Grossa-PR, São Gotardo-MG e Carandaí-MG e verificaram que o cultivar Brazlândia apresentou ampla adaptabilidade e comportamento previsível. Já Oliveira et al. (2008), avaliando diferentes épocas de semeadura de cultivares de cenoura, em distintos espaçamentos, também pela metodologia Eberhart \& Russel (1966), verificaram que, além de 'Brazlândia', os cultivares Alvorada e Nova Brasília também apresentaram comportamento favorável, em plantios em diferentes épocas e em distintos espaçamentos. Por sua vez, Silva et al. (2011, 2012), estudando a estabilidade e adaptabilidade de cenoura por técnicas multivariadas, verificaram que alguns genótipos apresentam adaptação geral (cv. BRS Planalto), enquanto outros apresentam adaptação específica (cv. Brasília), embora este último seja cultivado em todas as regiões produtoras de cenoura do País.

Diversos métodos para estudar a interação $\mathrm{G}$ x E foram propostos na literatura (Cruz \& Regazzi, 2001). Alguns são alternativos, enquanto outros podem ser utilizados conjuntamente com outros métodos, pois são complementares. Existem métodos baseados na análise de variância (Anova): tradicional, Plaisted \& Peterson (1959), Wricke (1965) e Annicchiarico (1992), e outros, baseados na regressão: Eberhart \& Russel (1966),
Finlay \& Wilkinson (1963) e Tai (1971). Outros, ainda, utilizam a regressão bissegmentada: Verma et al. (1978), Silva \& Barreto (1985) e Cruz et al. (1989). Há, também, métodos que utilizam análise não paramétrica (Huehn, 1990), análise visual (Lin \& Binns, 1988) e existem ainda aqueles baseados em análises de fatores (Murakami \& Cruz, 2004) ou em componentes principais (Zobel et al., 1988).

$\mathrm{Na}$ literatura, apesar da preferência por um ou outro método, a maioria dos trabalhos estudando a interação G x E são realizados pelos métodos de regressão, principalmente o de Eberhart \& Russel (1966), que, apesar de amplamente utilizado, apresenta algumas limitações, pois o índice ambiental é calculado em função das médias dos genótipos em cada ambiente. Isso resulta na não validade dos testes de hipótese, quando o número de ambientes e, ou, genótipos avaliados é pequeno (Cruz \& Regazzi, 2001).

Para resolver os problemas da metodologia de Eberhart \& Russel (1966), Cruz et al. (1989) propuseram uma modificação da análise de regressão bissegmentada, apresentada por Silva \& Barreto (1985), visando a torná-la mais simples e com propriedades estatísticas mais adequadas ao melhoramento genético. Por este método, os parâmetros de adaptabilidade são a média $\left(\hat{\mathrm{a}}_{0 \mathrm{i}}\right)$, a resposta linear aos ambientes desfavoráveis $\left(\hat{\mathrm{a}}_{\mathrm{li}}\right)$ e a resposta linear aos ambientes favoráveis $\left(\hat{\mathrm{a}}_{0 \mathrm{i}}+\hat{\mathrm{a}}_{1 \mathrm{i}}\right)$. A estabilidade é avaliada pelos quadrados médios dos desvios da regressão e pelo coeficiente de determinação $\left(\mathrm{R}^{2}\right)$.

Segundo Cruz \& Carneiro (2003), os métodos de determinação da estabilidade, da adaptabilidade e da estratificação ambiental são aplicados quase sempre de forma desassociada, sendo pouco úteis ao melhoramento genético. Assim, o melhorista sempre precisa tomar decisões sobre a seleção de determinado genótipo com base, em pelo menos, duas metodologias distintas. Uma metodologia que pode ser complementar a outras metodologias é a análise de fatores, proposta por Murakami \& Cruz (2004). Esta análise é baseada na técnica multivariada, por meio do princípio da similaridade de desempenho genotípico, que contempla estudos de adaptabilidade e estratificação ambiental.

A análise de fatores foi descrita por Johnson \& Wichern (1992). O princípio é fazer a rotação dos fatores e obter uma matriz de cargas fatoriais. Valores maiores ou iguais a 0,7 e de sinal positivo indicam ambientes com altas correlações dentro de cada fator, sendo possível estabelecer grupos. Valores entre 0,5 e 0,7 não garantem nenhuma definição na formação de grupos de ambientes, e valores abaixo de 0,5 indicam a impossibilidade de formação de grupos semelhantes de ambientes. A análise de estabilidade genotípica é realizada por 
meio de gráficos, por via de escores em relação aos fatores. São traçados eixos paralelos com a média dos escores, com formação de quatro quadrantes. No quadrante I, estão os genótipos de estabilidade ampla. Nos quadrantes II e IV, estão os genótipos com adaptabilidade específica à sub-região determinada pelo fator. Os genótipos localizados no quadrante III são os de baixo desempenho e passíveis de descarte. Esse método foi utilizado por Garbuglio et al. (2007), para a cultura do milho, e por Mendonça et al. (2007), para cultura da soja. Segundo esses autores, a análise de fatores é importante, pois além da estratificação ambiental, essa metodologia permite recomendar genótipos com adaptabilidade ampla ou para ambientes específicos.

O objetivo deste trabalho foi avaliar a estabilidade e adaptabilidade de oito genótipos de cenoura, em diferentes regiões do Brasil, por meio da análise de fatores e da regressão bissegmentada.

\section{MATERIAL E MÉTODOS}

Os experimentos foram conduzidos nos anos agrícolas 2009/10, 2010/11 e 2011/12, nas seguintes localidades: Setor de Campos Experimentais da Embrapa Hortaliças-Brasília-DF (CNPH), em sistema convencional (10CC, 11CC e 12CC) e orgânico (10CO, 11CO e 12CO); Núcleo Rural Rodeador, Brazlândia-DF, em sistema orgânico (10BO, 11BO e 12BO); São Gotardo-MG, em sistema convencional (10SG, 11SG e 12SG); Colônia Agrícola Lamarão-DF, em sistema orgânico (10MA e 11MA); Comunidade Belo Campo-Lapão-BA, em sistema convencional (10LA e 11LA) e Canoinhas-SC, em sistema convencional (12CA), totalizando 17 ambientes (Tabela 1). Em todos os ambientes foram avaliados oito genótipos de cenoura, sendo cinco populações (CNPH-598, CNPH-589, CNPH-600, CNPH-607 e CNPH-609) em fase de validação e três cultivares comerciais (Brasília, BRS Planalto e o híbrido Juliana).

Os experimentos foram instalados na segunda quinzena do mês de novembro de cada ano, até meados de janeiro do ano seguinte. Em todos os ambientes, a parcela experimental foi constituída de um canteiro com 1,0 m de comprimento por 1,0 m de largura. O sulco de plantio foi feito no sentido transversal ao canteiro em espaçamento duplo, sendo o espaçamento entre linhas simples de $10 \mathrm{~cm}$ e entre fileiras duplas de $20 \mathrm{~cm}$. O delineamento utilizado foi o de blocos ao acaso, com três repetições. O desbaste foi realizado aos 35 dias após o semeio, deixando um espaço entre plantas de $5 \mathrm{~cm}$, o que resultou numa população média equivalente a 890 mil plantas ha-1.

Os tratos culturais foram os mesmos das lavouras comerciais dos colaboradores (produtores de cenoura).

Os experimentos foram colhidos ao mesmo tempo em que foram realizadas as colheitas das áreas comerciais (110 dias após a semeadura). Foi avaliado o caráter massa de raízes com padrão comercial (MRC) e os valores, transformados para $\mathrm{t} \mathrm{ha}^{-1}$.

Todas as análises foram realizadas por meio do software Genes (versão 2009.7.0) (Cruz, 2006). Inicialmente, os dados foram submetidos às pressuposições da análise de variância (aditividade do modelo matemático, homogeneidade das variâncias entre os tratamen-

Tabela 1. Locais, anos agrícolas de avaliação e sistemas de cultivo de genótipos de cenoura para estudo de interação genótipos x ambientes

\begin{tabular}{|c|c|c|c|c|c|c|}
\hline \multirow{2}{*}{ Ambiente } & \multirow{2}{*}{ Local } & \multirow{2}{*}{$\begin{array}{c}\text { Ano } \\
\text { agrícola }\end{array}$} & \multirow{2}{*}{$\begin{array}{c}\text { Sistema } \\
\text { de cultivo }\end{array}$} & \multicolumn{3}{|c|}{ Coordenadas } \\
\hline & & & & $\mathbf{S}$ & $\mathbf{W}$ & Altitude (m) \\
\hline $10 \mathrm{BO}$ & Brazlândia-DF & $2009 / 10$ & Orgânico & $15^{\circ} 37^{\prime}$ & $48^{\circ} 04^{\prime}$ & 1.279 \\
\hline $11 \mathrm{BO}$ & Brazlândia-DF & 2010/11 & Orgânico & $15^{\circ} 37^{\prime}$ & $48^{\circ} 04^{\prime}$ & 1.279 \\
\hline $12 \mathrm{BO}$ & Brazlândia-DF & 2011/12 & Orgânico & $15^{\circ} 37^{\prime}$ & $48^{\circ} 04^{\prime}$ & 1.279 \\
\hline 10SG & São Gotardo-MG & $2009 / 10$ & Convencional & $19^{\circ} 28^{\prime}$ & $46^{\circ} 16^{\prime}$ & 1.197 \\
\hline $11 S G$ & São Gotardo-MG & 2010/11 & Convencional & $19^{\circ} 17^{\prime}$ & $46^{\circ} 06^{\prime}$ & 1.226 \\
\hline $12 \mathrm{SG}$ & São Gotardo-MG & 2011/12 & Convencional & $19^{\circ} 19^{\prime}$ & $46^{\circ} 09^{\prime}$ & 1.156 \\
\hline $10 \mathrm{MA}$ & Faz. Malunga/Brasília-DF & $2009 / 10$ & Orgânico & $15^{\circ} 58^{\prime}$ & $47^{\circ} 30^{\prime}$ & 903 \\
\hline $11 \mathrm{MA}$ & Faz. Malunga/Brasília-DF & 2010/11 & Orgânico & $15^{\circ} 58^{\prime}$ & $47^{\circ} 30^{\prime}$ & 903 \\
\hline $10 \mathrm{LA}$ & Lapão-BA & 2009/10 & Convencional & $11^{\circ} 30^{\prime}$ & $41^{\circ} 43^{\prime}$ & 792 \\
\hline $11 \mathrm{LA}$ & Lapão-BA & 2010/11 & Convencional & $11^{\circ} 30^{\prime}$ & $41^{\circ} 43^{\prime}$ & 792 \\
\hline $12 \mathrm{CA}$ & Canoinhas-SC & 2011/12 & Convencional & $26^{\circ} 11^{\prime}$ & $50^{\circ} 22^{\prime}$ & 799 \\
\hline $10 \mathrm{CC}$ & CNPH/Brasília-DF & 2009/10 & Convencional & $15^{\circ} 56^{\prime}$ & $48^{\circ} 09^{\prime}$ & 1.001 \\
\hline $11 \mathrm{CC}$ & CNPH/Brasília-DF & 2010/11 & Convencional & $15^{\circ} 56^{\prime}$ & $48^{\circ} 09^{\prime}$ & 1.001 \\
\hline $12 \mathrm{CC}$ & CNPH/Brasília-DF & 2011/12 & Convencional & $15^{\circ} 56^{\prime}$ & $48^{\circ} 09^{\prime}$ & 1.001 \\
\hline $10 \mathrm{CO}$ & CNPH/Brasília-DF & 2009/10 & Orgânico & $15^{\circ} 57^{\prime}$ & $48^{\circ} 08^{\prime}$ & 983 \\
\hline $11 \mathrm{CO}$ & CNPH/Brasília-DF & 2010/11 & Orgânico & $15^{\circ} 57^{\prime}$ & $48^{\circ} 08^{\prime}$ & 983 \\
\hline $12 \mathrm{CO}$ & CNPH/Brasília-DF & 2011/12 & Orgânico & $15^{\circ} 57^{\prime}$ & $48^{\circ} 08^{\prime}$ & 983 \\
\hline
\end{tabular}

Rev. Ceres, Viçosa, v. 61, n.6, p. 932-940, nov/dez, 2014 
tos, independência e normalidade dos erros) para cada ambiente. Como não ocorreu nenhuma restrição, realizou-se esta por ambiente e posteriormente a análise conjunta. Em seguida, realizaram-se o teste de agrupamento de Scott \& Knott (1974) e as análises, visando a estudar parâmetros de adaptabilidade e estratificação ambiental pelo método de Murakami \& Cruz (2004) e pela metodologia desenvolvida por Cruz et al. (1989), fundamentada na análise de regressão bissegmentada.

\section{RESULTADOS E DISCUSSÃO}

Ocorreram diferenças significativas para ambientes, para genótipos e para a interação genótipos x ambientes (dados não apresentados). O coeficiente de variação foi de 19,5\%, considerado satisfatório, diante da amplitude de ambientes testados. $\mathrm{O} \mathrm{R}^{2}$, que mede o ajuste do modelo, foi de 0,92 e mostrou-se adequado para este tipo de análise. A média geral dos experimentos foi de 31,57 $\mathrm{t} \mathrm{ha}^{-1}$, ou seja, semelhante à média nacional de produtividade, que está próxima de 30 t ha ${ }^{1}$ (Anuário Brasileiro de Hortaliças, 2012).

Pelo fato de ter sido significativa, desdobrou-se a interação genótipos x ambientes e realizou-se o teste de Scott \& Knott (1974), por meio do uso das médias ajustadas de genótipos (Tabela 2). O genótipo que apresentou o maior rendimento foi o cultivar 'Juliana', avaliado em 12CA $\left(96,30\right.$ tha $\left.^{-1}\right)$. O rendimento de 'Juliana' em 12SG (ambiente com intensiva utilização de insumos, principalmente fertilizantes, irrigação e agroquímicos) foi superior à produção dos cultivares e populações de polinização livre apenas na safra 2011/12. Ainda em relação a esse híbrido, nota-se que apresentou desempenho inferior, na safra 2010/11, comparado com o dos outros dois anos. Esse fato demonstrou a forte interação que os anos agrícolas podem exercer no desempenho de um cultivar, como, por exemplo, o excesso de chuvas. A capacidade de resposta com a melhoria do ambiente também pode ser observada com os cultivares/populações de polinização aberta. Esses genótipos, contudo, possuem capacidade inferior ao híbrido 'Juliana'. O aumento do rendimento de cultivares de polinização livre e híbridos, em regiões de intensificado uso de insumos, também é reportado por Oliveira et al.(2008). No trabalho desses autores, entretanto, os cultivares de polinização livre mostraram-se mais produtivos, em comparação com o híbrido utilizado como testemunha, demonstrando que nem sempre um híbrido é superior a um cultivar de polinização livre nesses sistemas produtivos.

Ressalta-se o desempenho do cultivar 'Brasília', que, embora tenha mais de 30 anos de mercado, apresenta alto rendimento de raízes comerciais, independentemente do ano e do local de avaliação. Esse fato explica o enorme sucesso desse cultivar, sendo hoje ainda muito cultivado em regiões como a de Irecê, no Estado da Bahia. Em vários ambientes, principalmente os de sistema orgânico, os desempenhos dos genótipos de cenoura não diferiram entre si, pelo teste de Scott-Knott (1974), a 5\% de probabilidade. Esse fato é comum, em experimentos de cenoura, na avaliação de genótipos com alto grau de melhoramento genético. Oliveira et al. (2008), avaliando cultivares de cenoura do grupo Brasília, em diferentes épocas de semeadura (de outubro a março) e dois espaçamentos, não encontraram diferenças entre esses cultivares para as condições de São José do Rio PardoSP, para a maioria dos caracteres relacionados a produtividade.

Na Tabela 3, encontram-se os resultados da análise de fatores para os oito genótipos de cenoura avaliados nos 17 ambientes. Nesse tipo de análise, o número de fatores finais utilizado é igual ao número de autovalores superiores às unidades existentes na matriz de correlações fenotípicas das variáveis padronizadas. Para se ter uma explicação satisfatória dos dados, é necessário, pela análise de fatores, que os autovalores maiores que um (1) sejam acima de $80 \%$. Nesse caso, consideraram-se os fatores 1, 2, 3 e 4 que explicaram $84,70 \%$ da variabilidade. Verificou-se que, após a rotação, o fator 1 indica a possibilidade de agrupamento dos ambientes $10 \mathrm{SG}$, 10CO, 10LA, 11LA e 12 CO. O fator 2 agrupou os ambientes $10 \mathrm{BO}, 11 \mathrm{CC}$ e $12 \mathrm{CC}$ e o fator 3 agrupou apenas 10MA, sem existir nenhuma razão específica para isso. Os ambientes 10CC, 11CC, 11MA, 11BO, 11SG, 12BO, $12 \mathrm{CA}$ e $12 \mathrm{SG}$ não foram agrupados por nenhum fator, pois suas cargas fatoriais sempre estiveram abaixo de $70 \%$.

Os ambientes 10LA e 11LA foram agrupados pelo fator 1 e indicam que Lapão -BA influenciou de maneira semelhante o comportamento produtivo nos dois anos agrícolas. Por sua vez, o fator 2 agrupou os ambientes 10BO, 11CC e 12CC, ou seja, três anos agrícolas, sistemas de cultivo diferentes, porém todos os experimentos conduzidos na mesma região ou localidade, BrasíliaDF. Esse fato demonstrou a interferência do ano agrícola no comportamento de genótipos de cenoura, pois diferentes anos e sistemas de cultivo podem conferir comportamento coincidente nos cultivares/populações de cenoura avaliados.

Como apenas os ambientes 10SG, 10CO, 10LA, $11 \mathrm{LA}$ e $12 \mathrm{CO}$ (fator 1) e 10BO, $11 \mathrm{CO}$ e $12 \mathrm{CC}$ (fator 2) foram os que indicaram possibilidade de agrupamento, pode-se avaliar a adaptabilidade dos genótipos para esses ambientes, por meio de um gráfico bidimensional, considerando os fatores 1 e 2 (Figura 1).

Verificou-se que no primeiro quadrante (Figura 1) ficaram localizados os genótipos Brasília, CNPH-589 e 


\begin{tabular}{|c|c|c|c|c|c|c|c|c|c|}
\hline \multirow[t]{2}{*}{ Ambientes } & \multicolumn{9}{|c|}{ Populaçóes/cultivares de cenoura } \\
\hline & CNPH-609 & CNPH-598 & CNPH-607 & CNPH-589 & CNPH-600 & Brasília & Juliana & BRS Planalto & Média Geral \\
\hline 10BO & $15,3 \mathrm{Ad}$ & $17,0 \mathrm{Ae}$ & $14,0 \mathrm{Ad}$ & $18,0 \mathrm{Ad}$ & $19,1 \mathrm{Ae}$ & $16,1 \mathrm{Ad}$ & $14,2 \mathrm{Ae}$ & $17,1 \mathrm{Ae}$ & 16,4 \\
\hline $10 \mathrm{CC}$ & $19,6 \mathrm{Bd}$ & $26,7 \mathrm{Ad}$ & $14,4 \mathrm{Bd}$ & $25,6 \mathrm{Ac}$ & $28,3 \mathrm{Ad}$ & $20,2 \mathrm{Bd}$ & $14,6 \mathrm{Be}$ & $32,5 \mathrm{Ad}$ & 22,7 \\
\hline $10 \mathrm{CO}$ & $11,0 \mathrm{Ad}$ & $15,1 \mathrm{Ae}$ & $8,8 \mathrm{Ad}$ & $11,4 \mathrm{Ad}$ & $16,2 \mathrm{Ae}$ & $16,0 \mathrm{Ad}$ & $11,2 \mathrm{Ae}$ & $12,9 \mathrm{Ae}$ & 12,8 \\
\hline $10 \mathrm{LA}$ & $26,6 \mathrm{Ac}$ & $25,6 \mathrm{Ad}$ & $28,7 \mathrm{Ac}$ & $29,9 \mathrm{Ac}$ & $35,5 \mathrm{Ad}$ & $34,7 \mathrm{Ac}$ & $32,8 \mathrm{Ad}$ & $32,3 \mathrm{Ad}$ & 30,8 \\
\hline $10 \mathrm{MA}$ & $24,4 \mathrm{Ac}$ & $25,1 \mathrm{Ad}$ & $25,2 \mathrm{Ac}$ & $25,8 \mathrm{Ac}$ & $28,6 \mathrm{Ad}$ & $23,4 \mathrm{Bd}$ & $22,1 \mathrm{Be}$ & $31,1 \mathrm{Ad}$ & 25,7 \\
\hline $10 \mathrm{SG}$ & $53,6 \mathrm{Aa}$ & $50,9 \mathrm{Ab}$ & $44,6 \mathrm{Bb}$ & $52,4 \mathrm{Aa}$ & $55,3 \mathrm{Ab}$ & $56,6 \mathrm{Aa}$ & $58,7 \mathrm{Ab}$ & $52,7 \mathrm{Ab}$ & 53,1 \\
\hline 11BO & $22,2 \mathrm{Ad}$ & $18,2 \mathrm{Ae}$ & $21,4 \mathrm{Ad}$ & $22,5 \mathrm{Ad}$ & $21,7 \mathrm{Ae}$ & $14,6 \mathrm{Ad}$ & $16,9 \mathrm{Ae}$ & $20,8 \mathrm{Ae}$ & 19,8 \\
\hline $11 \mathrm{CC}$ & $23,0 \mathrm{Bd}$ & $24,9 \mathrm{Ad}$ & $26,6 \mathrm{Ac}$ & $19,7 \mathrm{Bd}$ & $23,7 \mathrm{Be}$ & $19,6 \mathrm{Bd}$ & $22,2 \mathrm{Be}$ & $21,7 \mathrm{Be}$ & 22,7 \\
\hline $11 \mathrm{CO}$ & $35,8 \mathrm{Bc}$ & $39,7 \mathrm{Ac}$ & $33,0 \mathrm{Bc}$ & $36,7 \mathrm{Bc}$ & $39,5 \mathrm{Ac}$ & $39,5 \mathrm{Ab}$ & $29,8 \mathrm{Bd}$ & $37,1 \mathrm{Ac}$ & 36,4 \\
\hline $11 \mathrm{LA}$ & $23,8 \mathrm{Cd}$ & $31,9 \mathrm{Bd}$ & $28,0 \mathrm{Bc}$ & $26,0 \mathrm{Bc}$ & $29,9 \mathrm{Bd}$ & $37,4 \mathrm{Ab}$ & $30,4 \mathrm{Bd}$ & $32,8 \mathrm{Bd}$ & 30,0 \\
\hline $11 \mathrm{MA}$ & $16,1 \mathrm{Bd}$ & $21,2 \mathrm{Be}$ & $21,6 \mathrm{Bd}$ & $26,7 \mathrm{Ac}$ & $20,7 \mathrm{Be}$ & $15,3 \mathrm{Bd}$ & $19,4 \mathrm{Be}$ & $17,0 \mathrm{Be}$ & 19,8 \\
\hline 11SG & $41,7 \mathrm{Ab}$ & $44,3 \mathrm{Ac}$ & $46,6 \mathrm{Ab}$ & $46,7 \mathrm{Ab}$ & $41,4 \mathrm{Ac}$ & $43,4 \mathrm{Ab}$ & $39,3 \mathrm{Ac}$ & $40,5 \mathrm{Ac}$ & 43,0 \\
\hline $12 \mathrm{BO}$ & $20,7 \mathrm{Bd}$ & $25,4 \mathrm{Ad}$ & $20,6 \mathrm{Bd}$ & $25,8 \mathrm{Ac}$ & $26,6 \mathrm{Ad}$ & $29,2 \mathrm{Ac}$ & $20,6 \mathrm{Be}$ & $20,5 \mathrm{Be}$ & 23,7 \\
\hline $12 \mathrm{CA}$ & $61,2 \mathrm{Da}$ & $85,3 \mathrm{Ba}$ & $72,4 \mathrm{Ca}$ & $46,5 \mathrm{~Eb}$ & $75,7 \mathrm{Ca}$ & $53,6 \mathrm{Da}$ & $96,3 \mathrm{Aa}$ & $85,4 \mathrm{Ba}$ & 72,1 \\
\hline $12 \mathrm{CC}$ & $33,7 \mathrm{Bc}$ & $32,7 \mathrm{Bd}$ & $31,0 \mathrm{Bc}$ & $30,3 \mathrm{Bc}$ & $34,7 \mathrm{Bd}$ & $38,4 \mathrm{Ab}$ & $18,2 \mathrm{Ce}$ & $23,7 \mathrm{Ce}$ & 30,3 \\
\hline $12 \mathrm{CO}$ & $29,8 \mathrm{Bc}$ & $33,6 \mathrm{Bd}$ & $35,6 \mathrm{Bc}$ & $34,6 \mathrm{Bc}$ & $42,0 \mathrm{Ac}$ & $42,4 \mathrm{Ab}$ & $44,6 \mathrm{Ac}$ & $41,6 \mathrm{Ac}$ & 38,0 \\
\hline $12 S G$ & $38,1 \mathrm{Bb}$ & $38,6 \mathrm{Bc}$ & $42,2 \mathrm{Bb}$ & $45,5 \mathrm{Bb}$ & $45,0 \mathrm{Bc}$ & $49,7 \mathrm{Aa}$ & $55,1 \mathrm{Ab}$ & $42,5 \mathrm{BC}$ & 44,6 \\
\hline
\end{tabular}

${ }^{2}$ Médias seguidas de mesma letra 
CNPH-600, o que indica adaptabilidade ampla e que estes genótipos podem ser recomendados para os locais representados pelos fatores 1 e 2 (10SG, 10CO, 10LA, 11LA, $12 \mathrm{CO}, 10 \mathrm{BO}, 11 \mathrm{CC}$ e 12CC). Entre esses três genótipos é preferível a recomendação do cultivar Brasília, que apresentou maior produtividade (Tabela 2). A população CNPH-598 apresentou adaptação específica para o Distrito Federal, pois permaneceu no quadrante II e todos os ambientes (10BO, 11CO e 12CC) referemse a Brasília-DF. Os cultivares BRS Planalto e Juliana apresentaram adaptação aos ambientes 10SG, 10CO,
10LA, 11LA, 12 CO, ou seja, não foi possível afirmar com segurança a região e ao sistema de cultivo a que esses genótipos melhor se adaptam, pois, variam num mesmo local e sistema de cultivo, dependendo do ano agrícola.

A capacidade de agrupamento, ou não, de ambientes, pela análise de fatores foi relatada por Mendonça et al. (2007) e Garbuglio et al. (2007), em estudos de adaptabilidade com as culturas da soja e milho, respectivamente, em ensaios conduzidos na região sul do Brasil. No entanto, esses autores conseguiram agrupar, pela análi-

Tabela 3. Resumo da Análise de Fatores para oito genótipos de cenoura avaliados em 17 ambientes nos anos agrícolas de 2009/10, 2010/11 e 2011/12

\begin{tabular}{|c|c|c|c|c|c|c|c|c|c|}
\hline \multicolumn{3}{|c|}{ Estimativas de Autovalores } & \multirow{2}{*}{ Ambiente } & \multicolumn{6}{|c|}{ Cargas fatoriais após rotação } \\
\hline Raiz & (\%) Raiz & (\%) Acumulada & & Fator 1 & Fator 2 & Fator 3 & Fator 4 & $\mathrm{Com}^{\prime 1}$ & $\mathbf{I A}^{\prime 2}$ \\
\hline 5,42 & 31,90 & 31,90 & $10 S G$ & 0,76 & $-0,29$ & 0,00 & 0,19 & 0,70 & 21,27 \\
\hline 4,64 & 27,32 & 59,22 & $10 \mathrm{CC}$ & 0,33 & 0,67 & 0,61 & 0,04 & 0,93 & $-9,13$ \\
\hline 2,57 & 15,12 & 74,34 & $10 \mathrm{CO}$ & 0,81 & 0,45 & 0,02 & $-0,27$ & 0,93 & $-19,06$ \\
\hline 1,76 & 10,36 & 84,70 & $10 \mathrm{BO}$ & 0,41 & 0,78 & 0,32 & 0,28 & 0,96 & $-15,52$ \\
\hline 1,21 & 7,11 & 91,80 & 10MA & 0,10 & 0,49 & 0,79 & 0,05 & 0,88 & $-6,16$ \\
\hline 0,78 & 4,60 & 96,40 & $10 \mathrm{LA}$ & 0,80 & $-0,16$ & 0,11 & 0,31 & 0,77 & $-1,09$ \\
\hline 0,61 & 3,60 & 100,00 & $11 \mathrm{CC}$ & $-0,60$ & $-0,06$ & 0,22 & $-0,51$ & 0,67 & $-9,20$ \\
\hline 0,00 & 0,00 & 100,00 & $11 \mathrm{CO}$ & 0,40 & 0,89 & $-0,02$ & $-0,23$ & 1,00 & 4,57 \\
\hline 0,00 & 0,00 & 100,00 & $11 \mathrm{LA}$ & 0,79 & $-0,06$ & $-0,09$ & $-0,37$ & 0,77 & $-1,84$ \\
\hline 0,00 & 0,00 & 100,00 & $11 \mathrm{MA}$ & $-0,40$ & 0,28 & $-0,02$ & 0,65 & 0,65 & $-12,11$ \\
\hline 0,00 & 0,00 & 100,00 & $11 \mathrm{BO}$ & $-0,63$ & 0,40 & 0,44 & 0,37 & 0,90 & $-12,08$ \\
\hline 0,00 & 0,00 & 100,00 & $11 \mathrm{SG}$ & $-0,52$ & 0,50 & $-0,48$ & 0,19 & 0,78 & 11,06 \\
\hline 0,00 & 0,00 & 100,00 & $12 \mathrm{BO}$ & 0,61 & 0,57 & $-0,49$ & 0,07 & 0,94 & $-8,21$ \\
\hline 0,00 & 0,00 & 100,00 & $12 \mathrm{CA}$ & 0,06 & $-0,55$ & 0,61 & $-0,36$ & 0,81 & 40,19 \\
\hline 0,00 & 0,00 & 100,00 & $12 \mathrm{CC}$ & 0,09 & 0,72 & $-0,52$ & $-0,29$ & 0,88 & $-1,53$ \\
\hline 0,00 & 0,00 & 100,00 & $12 \mathrm{CO}$ & 0,77 & $-0,45$ & 0,21 & 0,15 & 0,86 & 6,15 \\
\hline 0,00 & 0,00 & 100,00 & $12 \mathrm{SG}$ & 0,57 & $-0,64$ & $-0,22$ & 0,43 & 0,97 & 12,69 \\
\hline
\end{tabular}

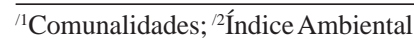

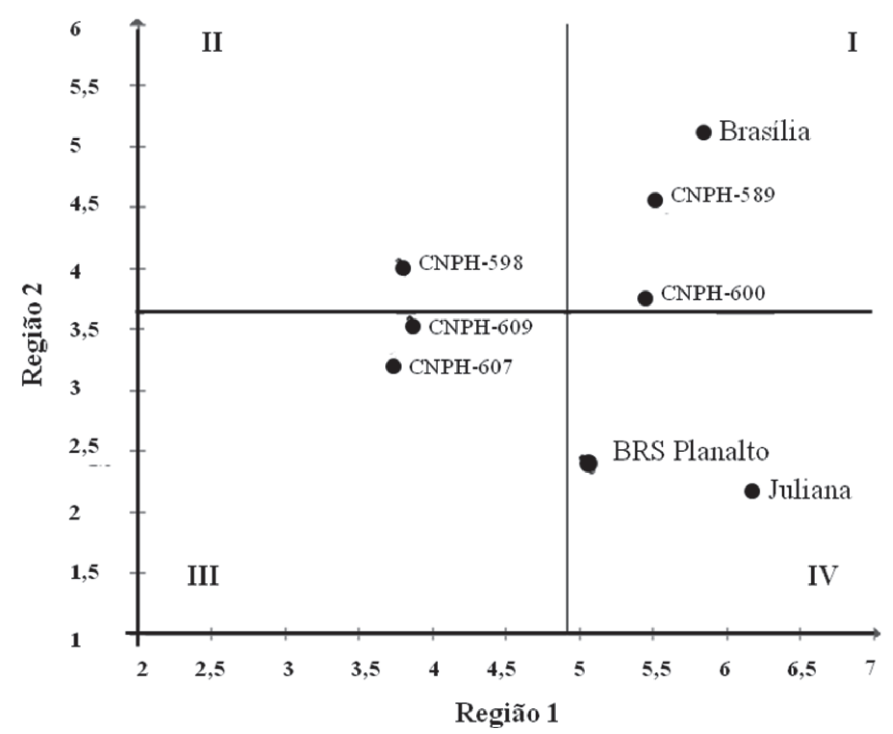

Figura 1. Plotagem dos escores, considerando os fatores 1 e 2, referentes aos resultados obtidos da análise de fatores para oito genótipos de cenoura avaliados em 17 ambientes nos anos agrícolas 2009/10, 2010/11 e 2011/12. 
se de fatores, a maioria dos ambientes avaliados, enquanto, neste trabalho, menos de $50 \%$ dos locais avaliados foram passíveis de agrupamento. Esse conflito de resultados pode ser explicado pela abrangência do território em que foram conduzidos os experimentos. Enquanto, nos experimentos dos referidos autores, os ensaios foram conduzidos, predominantemente, no Estado do Paraná, neste estudo, os ensaios foram realizados nas regiões nordeste, centro-oeste, sudeste e sul do Brasil, em ecossistemas distintos e sob diferentes sistemas de cultivo (orgânico e convencional).

$\mathrm{O}$ fato de os genótipos avaliados não serem indicados para todas as regiões produtoras contraria, possivelmente, o que se tem feito nos programas de melhoramento de cenoura do Brasil, ou seja, indicação de um cultivar para todas as regiões produtoras. A dificuldade de encontrar genótipos com ampla adaptabilidade é assunto indiscutível nos programas de melhoramento genético de grandes culturas. Tanto é assim que soja e milho, há muito tempo, possuem programas de melhoramento específicos para cada região de zoneamento agrícola.

As comunalidades para todos os ambientes apresentaram valores acima de $65 \%$, evidenciando alta eficiência na representação das variáveis por uma parte comum. Valores acima de $64 \%$ são tidos como satisfatórios, pois indicam correlação acima de $80 \%$ entre as variáveis padronizadas e da parte comum (Cruz \& Carneiro, 2003).

$\mathrm{O}$ índice ambiental indicou os ambientes 10SG, 11SG, $12 \mathrm{SG}, 12 \mathrm{CA}, 11 \mathrm{CO}$ e $12 \mathrm{CO}$ como favoráveis e os demais ambientes como desfavoráveis. Contudo, o fato de esses ambientes apresentarem índices positivos ou negativos não é, a priori, nenhum indicativo de correlação entre eles, por exemplo, 12CA e 12SG não tenderam a formar agrupamento com nenhum ambiente favorável ou desfavorável, como enfatiza Cruz \& Carneiro (2003).
Pela análise de estabilidade e adaptabilidade, método da regressão bissegmentada, proposta por Cruz et al. (1989), verificou-se que nenhum dos genótipos pode ser considerado ideal, pois esta metodologia preconiza que a média seja alta, $\hat{a}_{1 \mathrm{i}}$ significativamente menor que 1 , $\hat{\mathrm{a}}_{1 \mathrm{i}}+\hat{\mathrm{a}}_{2 \mathrm{i}}$ seja significativamente maior que 1 , e os desvios sejam não significativos. A dificuldade de encontrar o genótipo ideal também foi reportada em outros trabalhos na literatura, como os de Mendonça et al. (2007) e Backes et al. (2005). No entanto, os genótipos CNPH598 e BRS Planalto apresentaram-se como os mais promissores, para ambientes favoráveis, apresentando médias altas $\left(32,70\right.$ e 33, $07 \mathrm{t} \mathrm{ha}^{-1}$, respectivamente), $\hat{\mathrm{a}}_{1 \mathrm{i}}+\hat{\mathrm{a}}_{2 \mathrm{i}}$ $>1$ ( $<<0,01)$, desvio não significativo e $\mathrm{R}^{2}$ próximos de $95 \%$. Esses materiais, entretanto, não são indicados para ambientes desfavoráveis, uma vez que apresentaram $\hat{a}_{1 \mathrm{i}}$ não diferentes de 1. A boa estabilidade e adaptabilidade de BRS Planalto também são descritas por outros autores, demonstrando o bom desempenho desse cultivar (Silva et al. 2011; Silva et al., 2012).

A testemunha comercial 'Juliana' mostrou-se não adaptada a ambientes desfavoráveis (SG10, CO10, BO10, MA10, LA10, CC11, LA11, MA11, BO11, BO12 E CC12), com $\hat{a}_{1 \mathrm{i}}$ significativamente superior a 1 e baixa previsibilidade. Apresentou, porém, alta produtividade em ambientes favoráveis (CC10, CO11, SG11, CA12, CO12 E SG12) com produtividade de 53,94 tha-1 e alta adaptabilidade a esses ambientes ( $\left.\hat{\mathrm{a}}_{1 \mathrm{i}}+\hat{\mathrm{a}}_{2 \mathrm{i}}=1,70^{\prime \prime}\right)$. Verifica-se que entre ambientes favoráveis existem ambientes convencionais e orgânicos de cultivo. Esse fato demostra a capacidade de resposta dos híbridos tanto em sistemas de alta utilização de insumos como São Gotardo, mas também em sistemas orgânicos em que se utiliza adubação orgânica equilibrada e controle das doenças foliares com produtos aprovados para esse sistema de cultivo. A alta produtividade dos híbridos de ce-

Tabela 4. Estimativas de estabilidade e adaptabilidade para o caráter massa de raízes comerciais conforme a metodologia de Cruz et al. (1989) para oito genótipos de cenoura avaliados em 17 ambientes na safras 2010, 2011 e 2012

\begin{tabular}{|c|c|c|c|c|c|c|c|}
\hline \multirow{2}{*}{ Genótipos } & \multicolumn{3}{|c|}{ Média nos ambientes } & \multirow{2}{*}{$\beta_{1 \mathrm{i}}$} & \multirow{2}{*}{$\beta_{1 \mathrm{i}}+\boldsymbol{\beta}_{2 \mathrm{i}}$} & & \multirow{2}{*}{$\mathbf{R}^{2}(\%)$} \\
\hline & Geral & Desf. & Fav. & & & & \\
\hline CNPH-609 & 29,20 & 21,48 & 43,35 & $0,89^{\text {ns }}$ & $0,84^{\mathrm{ns}}$ & $35,25^{\mathrm{ns}}$ & 94,38 \\
\hline CNPH-598 & 32,70 & 23,97 & 48,70 & $0,99^{\text {ns }}$ & $1,38^{\prime \prime}$ & $39,27^{\mathrm{ns}}$ & 95,91 \\
\hline CNPH-607 & 30,27 & 21,83 & 45,73 & $0,98^{\mathrm{ns}}$ & $1,02^{\mathrm{ns}}$ & $42,10^{\text {ns }}$ & 94,68 \\
\hline CNPH-589 & 30,85 & 23,78 & 43,80 & $0,81 "$ & $0,30^{\prime \prime}$ & $49,02^{\mathrm{ns}}$ & 89,04 \\
\hline CNPH-600 & 34,36 & 25,91 & 49,87 & $0,98^{\mathrm{ns}}$ & $1,04^{\mathrm{ns}}$ & $11,22^{\mathrm{ns}}$ & 98,54 \\
\hline 'Brasília' & 32,31 & 24,06 & 47,44 & $1,00^{\mathrm{ns}}$ & $0,40^{\prime \prime}$ & $71,76^{*}$ & 89,57 \\
\hline 'Juliana' & 32,13 & 20,24 & 53,94 & $1,31 "$ & $1,70^{\prime \prime}$ & $86,71^{* *}$ & 94,64 \\
\hline 'BRS Planalto' & 33,07 & 23,85 & 49,97 & $1,05^{\mathrm{ns}}$ & $1,34^{\mathrm{ns}}$ & $57,75^{\mathrm{ns}}$ & 94,40 \\
\hline
\end{tabular}

\footnotetext{
, , ' : Significativamente diferente de um, pelo teste t, a 5 e $1 \%$ de probabilidade, respectivamente;

*,**: Significativamente diferente de zero, pelo teste $\mathrm{F}$, a 5 e $1 \%$ de probabilidade, respectivamente;

ns: não significativo. $\beta_{1 \mathrm{i}}$ : média geral do cultivar $\mathrm{i} ; \beta_{2 \mathrm{i}}$ : coeficiente de regressão linear associado a ambientes desfavoráveis; $\beta_{1 \mathrm{i}}+\beta_{2 \mathrm{i}}$ : coeficiente de regressão linear associado a ambientes favoráveis; variância dos desvios da regressão; $\mathrm{R}^{2}$ : coeficientes de determinação.
} 
noura, em regiões de alta intensidade de uso de insumos agrícolas como São Gotardo ou em sistemas orgânicos tecnificados, podem dar retorno financeiro aos produtores, amortizando o gasto adicional com sementes híbridas.

O cultivar tradicional de cenoura de verão Brasília apresentou alta produtividade em ambientes favoráveis $\left(47,44 \mathrm{t} \mathrm{ha}^{-1}\right)$. Porém, pouco adaptada a ambientes desfavoráveis ou favoráveis, $\hat{a}_{1 \mathrm{i}}=1,00^{\mathrm{ns}}$ e $\hat{\mathrm{a}}_{1 \mathrm{i}}+\hat{\mathrm{a}}_{2 \mathrm{i}}=0,40^{\prime \prime}$, respectivamente. Além disso, apresentou baixa previsibilidade $=71,76^{*}$, ou seja, seu comportamento foi não coincidente, nos diferentes ambientes de avaliação. O comportamento instável de 'Brasília' também foi descrito por Oliveira et al. (2008), na avaliação da estabilidade de cultivares de cenoura, em diferentes épocas de semeadura e densidades populacionais, em cultivo de cenoura de verão, no Estado de São Paulo, e por Silva et al. (2011; 2012), na avaliação de genótipos de cenoura em diferentes regiões brasileiras. No entanto, Silva et al. (2012) destacam o alto desempenho de 'Brasília', em Irecê, no Estado da Bahia, sendo atualmente o cultivar mais plantado nessa região. De modo semelhante ao dos resultados deste estudo, os genótipos com maior grau de melhoramento genético, como 'Alvorada' e 'Brazlândia', foram os mais indicados por esses autores.

Neste estudo, o cultivar Brasília não apresentou comportamento estável nem adaptado ao conjunto de ambientes avaliados. Contudo, esse cultivar é considerado um marco no melhoramento vegetal no Brasil, pois possibilitou o plantio de cenoura em períodos em que os cultivares tradicionais, de inverno, não se desenvolviam por causa do calor e do ataque da queima-das-folhas. Além disso, esse cultivar é fonte direta de germoplasma da maioria dos cultivares modernos de cenoura de verão. A maior estabilidade e adaptabilidade das populações derivadas de 'Brasília' é resultado das inúmeras seleções dentro dessa população, que permitiram selecionar indivíduos com maior resposta frente às mudanças de ambiente de cultivo e região.

As populações CNPH-600, CNPH-607 e CNPH-609 não apresentaram adaptação a ambientes favoráveis ou desfavoráveis. CNPH-600, no entanto, apresentou má dia geral alta $\left(34,36 \mathrm{t} \mathrm{ha}^{-1}\right)$ e alta previsibilidade $\left(\hat{\sigma}_{g i}^{2}\right.$ $\left.=11,22^{\text {ns }}\right)$, podendo ser indicado como cultivar. O genótipo ideal, segundo a metodologia de Cruz et al. (1989), não foi encontrado no conjunto de genótipos estudados. Resultados semelhantes foram encontrados por Hamawaki \& Santos (2003), Vendruscolo et al. (2001), Garbuglio et al. (2007) e Mendonça et al. (2007), com as culturas do milho, milho pipoca, milho e soja, respectivamente. Para o parâmetro estabilidade, seria pouco provável encontrar o genótipo ideal, pois os programas de melhoramento procuram desenvolver cultivares responsivos à melhoria do ambiente, compensando, assim, os maiores gastos com a intensificação do uso de insumos agrícolas.

Os estudos da adaptabilidade e estabilidade sempre precisam ser analisados por mais de uma metodologia, para que o pesquisador possa ter maior embasamento dos seus resultados, para a recomendação de cultivares. Segundo Cruz \& Regazzi (2001), existem mais de dez diferentes metodologias para o estudo de estabilidade e adaptabilidade. A escolha de um ou outro método depende do número de ambientes disponíveis, da precisão e do tipo de informação desejadas. Além do mais, alguns métodos são alternativos, enquanto outros são complementares, podendo ser usados conjuntamente.

A análise de fatores permite identificar os genótipos com adaptação ampla, adaptação restrita a ambientes favoráveis ou desfavoráveis e não adaptados. No entanto, a recomendação é feita com base apenas nos ambientes passíveis de agrupamento. Neste estudo, apenas oito dos 17 ambientes foram agrupados, ou seja, 47\%. Desse modo, não é possível fazer nenhuma inferência sobre o comportamento dos genótipos avaliados nos ambientes não agrupados. Por outro lado, a regressão bissegmentada é mais ampla e classifica os genótipos com base no seu comportamento médio, considerando todos os ambientes avaliados. Contudo, para esse estudo e por essa metodologia, nenhum genótipo foi classificado como ideal, ou seja, com média geral alta, estável em ambientes desfavoráveis, mas com alta capacidade de resposta em ambientes favoráveis e com alta previsibilidade.

A dificuldade de recomendar genótipos de cenoura com adaptação ampla para as regiões avaliadas neste trabalho era prevista, haja vista a distância geográfica e as condições distintas em que os genótipos foram avaliados. No caso de cenoura, os programas de melhoramento estão menos avançados do que os das grandes culturas; por isso, os melhoristas ainda precisam lançar um novo cultivar, com base no comportamento médio que ele apresente nos diferentes locais e anos de avaliação, deixando, assim, de capitalizar os efeitos intrínsecos de cada ambiente, na seleção de genótipos de adaptabilidade específica.

Merece destacar que, pela análise de fatores, o cv. Brasília apresentou adaptação ampla, considerando-se os ambientes 10SG, 10CO, 10LA, 11LA, 12CO, 10BO, $11 \mathrm{CC}$ e $12 \mathrm{CC}$. Por outro lado, não foi recomendado pela regressão bissegmentada. No entanto, esse cultivar é até hoje produzido nas mais distantes localidades do território nacional, com satisfatória produtividade de raízes comerciais (Silva et al., 2012). Com base apenas em critérios metodológicos de estabilidade e adaptabilidade, esse cultivar, provavelmente, não teria sido lançado 
e a cadeia produtiva da cenoura não se teria beneficiado dos impactos positivos desse cultivar, ou seja, a experiência do melhorista, as oportunidades de mercado e o conhecimento acerca dos pré-cultivares que estão sendo avaliados também são critérios importantes na tomada de decisão do lançamento de um novo cultivar.

\section{CONCLUSÕES}

Nenhum genótipo apresentou-se adaptado ou estável para o conjunto de ambientes testados pela análise de fatores e análise de regressão bissegmentada.

Os resultados obtidos por essas metodologias demonstram a dificuldade de recomendação de um cultivar de cenoura para todas as regiões produtoras e que programas regionalizados devem ser iniciados.

A utilização dos dois métodos associados permite tomada de decisão mais segura, acerca do comportamento dos genótipos de cenoura.

\section{REFERÊNCIAS}

Annicchiarico P (1992) Cultivar adaptation and recommendation from alfafa trials in Northern Italy. Journal of Genetics \& Breeding, 46:269278.

Anvisa - Agência Nacional de Vigilância Sanitária (2011) Programa de Análise de Resíduos de Agrotóxicos em Alimentos: Relatório de Atividades de 2010 Disponível em: <http://portal.anvisa.gov.br/ wps/wcm/connect/b380fe004965d38ab6abf74ed75891ae/ Relat\%C3\%B3rio+PARA+2010+-+Vers\%C3\%A3o +Final.pdf?MOD= AJPERES>. Acessado em: 23 de setembro de 2013.

Anuário Brasileiro de Hortaliças (2012) Brazilian Vegetable Yearbook. Santa Cruz do Sul, Gazeta. 88p.

Backes RL, Elias HT, Hemp S \& Nicknich W (2005) Adaptabilidade e estabilidade de genótipos de feijoeiro no Estado de Santa Catarina. Maringá, 27:309-314.

Cargnin A, Souza MA, Carneiro PCS \& Sofiatti V (2006) Interação entre genótipos e ambientes e implicações em ganhos com seleção em trigo. Pesquisa Agropecuária Brasileira, 41:987-993.

Cruz CD \& Regazzi AJ (2001) Modelos biométricos aplicados ao melhoramento genético. $2^{\mathrm{a}}$ ed. Viçosa, UFV. 390p.

Cruz CD (2006) Programa Genes - Biometria. Viçosa, Editora UFV. 382p.

Cruz CD \& Carneiro PCS (2003) Modelos biométricos aplicados ao melhoramento genético. Viçosa, UFV. 585p.

Cruz CD, Torres RA \& Vencovsky R (1989) An alternative approach to the stability analysis proposed by Silva e Barreto. Revista Brasileira de Genética, 12:567-580.

Eberhart SA \& Russel WA (1966) Stability parameters for comparing varieties. Crop Science, 6:36-40.

Finlay KW \& Wilkinson GN (1963) The analysis of adaptation in a plant breeding programme. Australian Journal of Agricultural Research, 14:742-754.

Garbuglio DD, Gerage AC, Araújo PM, Fonseca Junior NS \& Shioga PS (2007) Análise de fatores e regressão bissegmentada em estudos de estratificação ambiental e adaptabilidade em milho. Pesquisa Agropecuária Brasileira, 42:183-191.
Hamawaki OT \& Santos PG (2003) Adaptabilidade e estabilidade de genótipos de milho avaliadas por meio do modelo de regressão. Ciência Rural, 33:195-199.

Huehn M (1990) Nonparametric measures of phenotypic stability. Part I: Theory Euphytica, 47:189-194.

Johnson RA \& Wichern DW (1992)Applied multivariate statistical analysis. $3^{\mathrm{a}}$ ed. New Jersey, Prantice Hall. 642p.

Lin CS \& Binns MR (1988) A superiority measure of cultivar performance for cultivar x location data. Canadian Journal of Plant Science, 68:193198.

Mendonça O, Carpentieri-Pípolo V, Garbuglio DD \& Fonseca Junior NS (2007) Análise de fatores e estratificação ambiental na avaliação da adaptabilidade e estabilidade em soja. Pesquisa Agropecuária Brasileira, 42:1567-1575.

Murakami DM \& Cruz CD (2004) Proposal of methodologies for environment stratification and analysis of genotype adaptability. Crop Breeding and Applied Biotechnology, 4:07-11.

Oliveira CD, Braz LT \& Banzatto DA (2008) Adaptabilidade e estabilidade fenotípica de cultivares de cenoura. Horticultura Brasileira, 26:8892.

Oliveira CD, Braz LT \& Banzatto DA (2005) Adaptabilidade e estabilidade fenotípica de genótipos de cenoura. Horticultura Brasileira, 23:743748 .

Plaisted RL \& Peterson LC (1959) A technique for evaluating the ability of selections to yield consistently in different locations and seasons. American Potato Journal, 36:381-385.

Scott AJ \& Knott MA (1974) A cluster analysis method for grouping means in the analysis of variance. Biometrics, 30:507-512.

Silva GO, Carvalho ADF, Vieira JV \& Benin G (2011) Verificação da adaptabilidade e estabilidade de populações de cenoura pelos métodos AMMI, GGE biplot e REML/BLUP. Bragantia, 70:494-501.

Silva GO, Carvalho ADF, Vieira JV \& Fritsche-Neto R (2012) Adaptabilidade e estabilidade de populações de cenoura. Horticultura Brasileira, 30:80-83.

Silva JGC \& Barreto JN (1985) Aplicação da regressão linear segmentada em estudos da interação genótipo x ambiente. In: $1^{\circ}$ Simpósio de experimentação agrícola, Piracicaba. Anais, ESALQ. p.49-50.

Tai GCC (1971) Genotype stability analysis and its application to potato regional trials. Crop Science, 11:184-190.

Vendruscolo ECG, Sacapin CA, Pacheco CAP, Oliveira VR, Braccini AL \& Gonçalves-Vidigal MC (2001) Adaptabilidade e estabilidade de cultivares de milho-pipoca na região centro-sul do Brasil. Pesquisa agropecuária Brasileira, 36:123-130.

Verma MM, Chahal GS \& Murty BR (1978) Limitations of conventional regression analysis: a proposed modification. Theoretical and Applied Genetics, 53:89-91.

Wricke G (1965) Zur berechnung der okovalenzbeisommerweizen und hafer. Z. Pflanzenzuchtg, 52:127-138.

Zobel RW, Wright MJ \& Gauch HG (1988)Statistical analysis of a yield trial. Agronomy Journal, 80:388-393.

Rev. Ceres, Viçosa, v. 61, n.6, p. 932-940, nov/dez, 2014 